\title{
De cómo escribir un artículo de divulgación y no matar de aburrimiento a tus lectores
}

\author{
Guillermo N. Murray-Tortarolo
}

\begin{abstract}
Resumen
Una de las lecturas más bellas del mundo es la divulgación de la ciencia bien hecha. Es interesante, dinámica, divertida y te enseña cosas nuevas del mundo donde vives. Pero escribir artículos con todas esas características es un reto, para muchos, apabullante. Al enfrentarnos a la hoja en blanco nos atoramos y nuestras ideas no son plasmadas en el papel como nos gustaría. ¿Alguna vez te ha pasado? Te sientas para escribir un ensayo, un trabajo o hasta una carta de amor..., y aunque las ideas están en tu cabeza, al final la tinta se resiste. Aquí te traigo la medicina para este bloqueo. A lo largo de las siguientes páginas te platicaré mi experiencia a la hora de escribir, para que puedas hacer frente a la página y llenar las cuartillas con historias de divulgación que se parezcan a lo que estabas pensando.
\end{abstract}

Palabras clave: divulgación de la ciencia, método literario, fondo y forma, bloqueo de escritor.

\section{ON HOW TO WRITE SCIENCE FOR A GENERAL PUBLIC AND NOT SMOTHER YOUR}

\section{READERS BY BOREDOM}

\begin{abstract}
One of the most beautiful things in this wide world is reading science that is well written and reported. It is interesting, dynamic, fun and it teaches you novel facts about our universe. But writing a manuscript with all those characteristics is a remarkable challenge. We get stuck while facing the blank page and our ideas are rarely inked the way we would have liked. Have you ever been in such a situation? Sitting down, ready to write an essay, a manuscript or even a love letter..., and, although you have all the ideas in your mind, they simply refuse to be placed on paper. Here, I bring you a remedy against scientific writing block. On the next few pages I will tell you a bit about my writing experience, so you can face the page and write texts that look a lot closer to what you were thinking.
\end{abstract}

Keywords: science outreach, writing methods, substance and structure, writer's block. 


\section{Guillermo N. Murray-Tortarolo}

Es Investigador Asociado C en el Instituto de Investigaciones en Ecosistemas y Sustentabilidad de la Universidad Nacional Autónoma de México (UNAM). Su tema principal de trabajo es la investigación ecológica a distintas escalas espacio-temporales. Le interesa entender los impactos del cambio y la variación climática sobre distintos procesos ecológicos y agronómicos de México y el mundo. También tiene un profundo interés y pasión por la divulgación de la ciencia y por la enseñanza de la misma.

No hay nada más terrible que estar sentado frente a una página en blanco con una cabeza llena de ideas. Creo que nos ha pasado a más de uno; durante días rumiamos y pensamos una idea - una historia - y todas sus diferentes vertientes, pero cuando llegamos a la computadora (o en su defecto a la máquina de escribir para mis lectores hípster o de la tercera edad) no salen las letras. Toda nuestra grandiosa imaginación se queda atorada y lo poco que escribimos no se parece en nada a lo que habíamos pensado. Así nos pasa con los trabajos escolares, los ensayos, los resúmenes..., bueno, a veces hasta con las cartas de amor; y si se trata de divulgación de la ciencia ni se diga, los datos, los números, las interpretaciones, todo se hace una maraña que acaba por frustrarnos y enojarnos pues no representa lo que queremos decir.

Algunos entonces recurrimos a la otra estrategia: vomitar todo lo que hay en nuestra cabeza sin ningún orden. Llenamos páginas y páginas de cifras e ideas sin forma, que difícilmente se leen como una historia. No es de sorprenderse que cuando entregamos estos trabajos en la escuela o un artículo de divulgación a una revista, éstos sean rechazados inmediatamente - o que nos reprueben-. Claro, esto da aún más coraje porque sabemos que tenemos la historia, que tenemos las ideas y que es interesante, lo que nos atora es cómo darle forma frente a la página en blanco.

¿Entonces, qué podemos hacer? En las siguientes páginas te voy a transmitir un poco de mi experiencia como divulgador de la ciencia y narrador de historias. Trataré de explicarte cómo es mi proceso creativo y cómo transmito las ideas desde mi cabeza hasta el papel, para que tengan estructura, una narrativa coherente, un trasfondo interesante y, ¿por qué no?, hasta un poco de sentido del humor. Al final, lo más importante de todo —el mensaje que espero te lleves luego de acompañarme por estas páginas - es que para escribir (cualquier cosa) lo más importante es reescribir. 


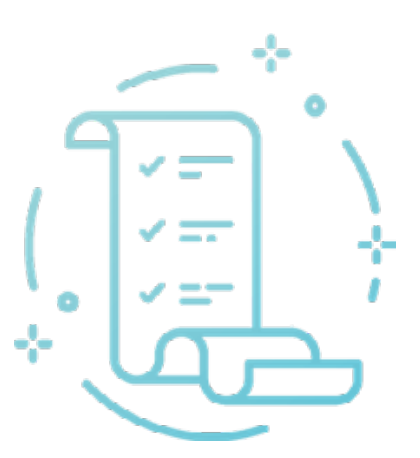

Para comenzar, vamos a ver los tres ingredientes fundamentales que conforman cualquier texto y que nos ayudarán a escribir un artículo de divulgación (y cualquier texto en general): el fondo -la historia-, la forma - la estructura- y el flujo -o ritmo-. Empecemos, entonces, a explicar cada uno de ellos.

\section{El primer ingrediente: la historia que quieres contar}

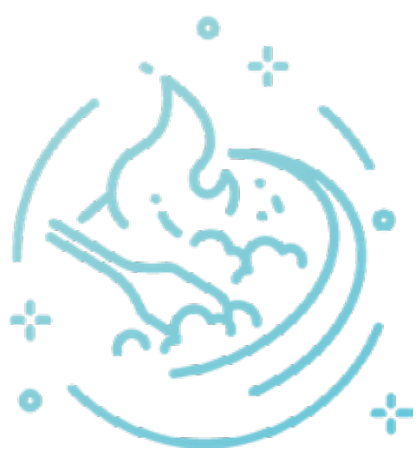

Lo más importante de todo - la carne de los tacos, el merengue del pastel_-, es comenzar por una buena historia. Lo bueno es que en ciencia abundan las historias, y a lo largo de nuestra formación en la licenciatura o el posgrado, habrá muchísimas cosas que nos interesen y nos apasionen. De allí es donde tenemos que partir, de algo que nos llene de júbilo cuando lo leemos, lo platicamos o lo trabajamos.

Una vez que elegimos el tema, lo siguiente es empezar a estudiar al respecto. Lo más importante es tratar de entender los distintos matices de la historia y construir un sistema solar argumental, pero ¿qué significa esto? Decidir qué elementos de la historia forman parte central de la misma (el sol), cuales son secundarios (planetas), terciarios (las lunas) y cuáles de plano, no podemos incluir —aunque muchas veces nos encanten-.

Les doy un ejemplo: el impacto del cambio climático sobre los arrecifes de coral. Este es un tema que a muchos nos apasiona, pues los corales son núcleos globales de biodiversidad que se ven amenazados por el aumento de temperatura del mar. Pensemos en todos los elementos de la historia. En términos de los corales: el cómo se forman, su importancia ecológica y biológica, las zonas donde existen..., bueno, hasta los mejores lugares para ir a bucear. En términos del cambio climático, los impactos: humano, sobre los ecosistemas,

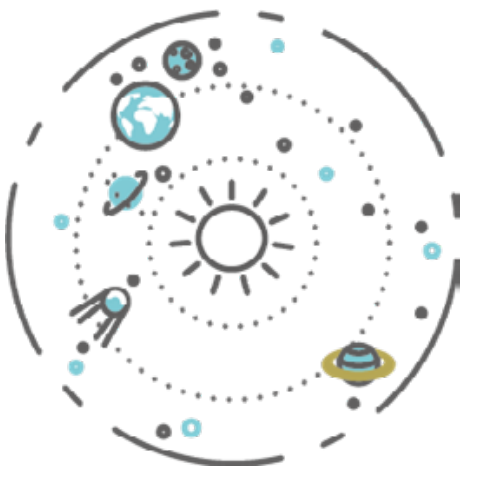
económico; los acuerdos globales, por qué el gobierno de euA pretende que no existe el cambio climático, hasta si es una cuestión de creencia o no. Finalmente, el aumento en la temperatura oceánica: si es normal, si ha ocurrido antes, por qué ocurre... y si esto significa que podré irme a la playa en invierno y va a estar bien agradable para nadar. Todos estos son elementos de la historia y, dependiendo como la queramos contar, tendrán un orden jerárquico diferente en nuestro 
sistema solar narrativo. Es posible que, si la historia se centra en el impacto del cambio climático en los arrecifes de coral, el Sol estructural sea la formación de dichos ecosistemas y su dependencia a la temperatura; los planetas serán los argumentos relacionados a cómo el cambio climático ha alterado la temperatura; y las lunas, las acciones que podemos llevar a cabo para remediarlo. Otras cosas, como la política pública de EUA o los mejores lugares turísticos para ver el coral, deben quedar fuera de la historia por más interesantes que sean.

En el idioma castellano (y en casi todos) los sistemas solares narrativos tienen otro nombre: la estructura de la oración. Si, así como lo repetimos desde la primaria: sujeto, verbo y predicado. El comprender dicha estructura dentro de la historia que queremos contar es el primer paso para generar un hilo lógico e interesante en nuestra narrativa (y no irnos por las nubes). Entonces, siempre de los siempres, antes de sentarte a escribir, debes de preguntarte: ¿a quién le pasa la historia? El sujeto, el Sol: los arrecifes de coral. ¿Qué pasa en la historia? El verbo, Ios planetas: el aumento de temperatura. ¿Cuáles son los detalles? El predicado, las lunas: el cambio climático y qué podemos hacer para remediarlo.

\section{El segundo ingrediente: la estructura de la historia}

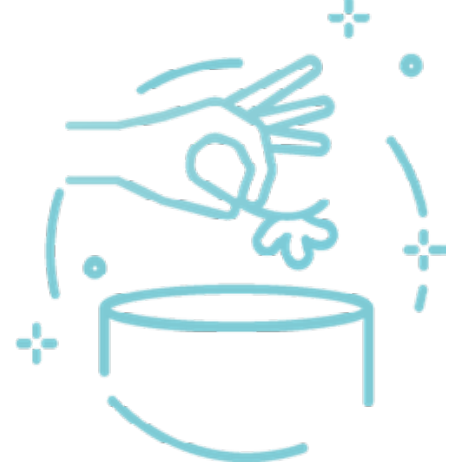

Pero que son los tacos sin una buena salsa y verdura. Así también, el tener una historia que nos guste o nos apasione y que hayamos estudiado no siempre es garantía de que podamos contarla de manera amena, de transformarla en una buena historia. Ésta es la parte donde el método literario juega un rol fundamental.

Así como la carne de nuestra historia se basa en un sujeto, verbo y predicado, así también la estructura. Para que un texto tenga sentido, la forma de nuestro lenguaje se debe repetir en cada una de sus partes. Quiero decir que cada oración debe tener sujeto, verbo y predicado, pero así también cada párrafo, cada sección y el texto completo. Toma como ejemplo este artículo, el texto abre presentando al problema (sujeto, la divulgación), en las siguientes tres secciones se desarrollan las acciones (verbo, el método) y en el cierre se dan recomendaciones generales (predicado, algunos detalles). Y así también sucede al interior de cada sección particular y para cada párrafo. Esta estructura facilita que tus lectores puedan seguir las ideas y saber de lo que les estás hablando. Y una recomendación particular, siempre comienza los párrafos con la idea central de lo que vas a hablar en dicho párrafo y trata de centrar los mismos a una sola idea.

El segundo factor fundamental para contar de manera amena la historia es saber a quién será dirigida. Y es que una parte fundamental del escribir es acordarse de que no estás escribiendo para ti mismo, sino para otros. Y no es lo mismo si te van a leer chavos de secundaria, de posgrado, tu abuelita o tu 
director de tesis. Conocer a tu audiencia es fundamental para poder escribir al nivel adecuado, con el vocabulario correcto y la extensión necesaria.

Si tomamos por ejemplo este artículo, todo el tiempo tuve presente que se trata de jóvenes de licenciatura y a lo mejor uno que otro de la prepa, con interés en escribir divulgación. Al saber esto, puedo dar por sentado que todos han tenido experiencias similares en escribir ensayos, resúmenes y hasta tesis o tesinas, y que han leído, escuchado o visto algo de las distintas áreas de la ciencia, de modo que estructuren el texto acorde. Si se tratara de cómo escribir textos históricos en la carrera de Historia de la UNAM, el resultado hubiera sido completamente diferente. Si algo te tiene que quedar de todo esto es: siempre recuerda a tu audiencia y que el texto no es para ti.

\section{El tercer ingrediente: el ritmo}

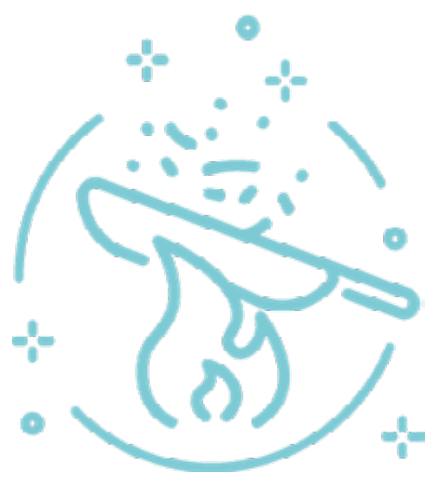

El ingrediente final para armar una historia genial es el ritmo, el flujo o, como lo llaman los raperos, el flow. Volviendo al ejemplo de los tacos, al final, en el millón de puestos en México, los tacos están conformados por carne, tortillas, verduras y salsa; pero lo que hace únicos a algunos puestos, lo que hace que estemos dispuestos a formarnos una hora, es la sazón particular de cada uno. Así también a la hora de escribir, es el ritmo personal lo que le da sabor a la historia. Es lo que hace que García Márquez sea García Márquez y Saramago, Saramago.

Es en este último ingrediente donde se devela tu personalidad y muchas veces tu estado de ánimo con respecto a la historia. ¿Te sientes con ganas de escribir algo divertido?, ¿o el tema que quieres abordar necesita seriedad? ¿Qué tipo de metáforas puedes emplear? En fin, se trata de poner tu corazón en el texto. En general, en mi experiencia, la divulgación de la ciencia es mucho mejor si la haces divertida y amena (tanto escrita como oral). Trata de darle algo de sentido del humor, identifícate con el lector y dale elementos de su cotidianidad (como los tacos) y no tengas miedo de experimentar con las palabras.

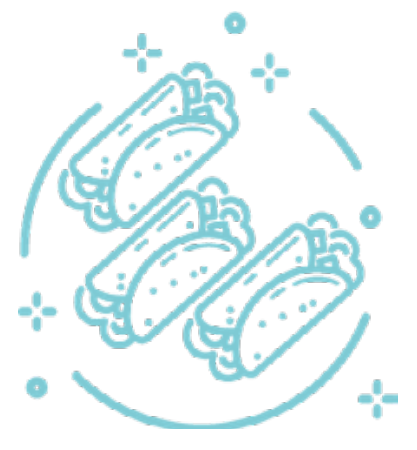




\section{Algunos comentarios finales}

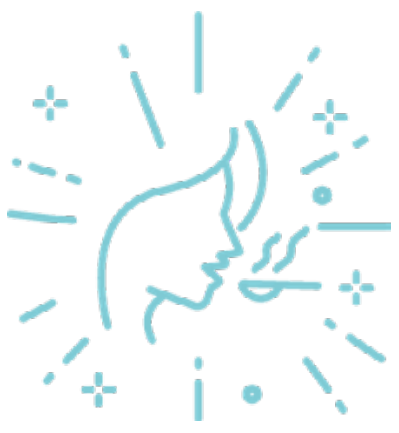

Ahora ya tienes la receta para escribir divulgación (o hacer unos tacos sabrosos..., ya no supe cual), pero hay un par de cosas más que quiero decirte. En primer lugar, la forma más sencilla de asegurarte que tu texto es lógico, se puede seguir, es interesante y divertido, es dárselo a otros para que lo lean. Entre más variado sea tu público mejor: dáselo a tu mamá, a tus amigos, a tu abuelita, al profe y hasta a tus enemigos. Entre más variados sean los comentarios, mejor. Trata de mantenerte receptivo a la crítica, siempre recordando que están juzgando tu texto y no a ti (aunque a veces sintamos que los escritos

son parte de nosotros, hay que tratar de separarse de ellos). Todos los textos se benefician de otras voces y opiniones.

Finalmente quiero agregar que, si bien hemos acotado los tres ingredientes de la divulgación de la ciencia, en realidad se trata de una receta que sirve para redactar casi cualquier texto. La misma estructura la puedes usar para los ensayos, los resúmenes y hasta la escritura de artículos científicos. Siempre recordando que escribir bien es reescribir. Al final el aprender a plasmar las ideas en papel, el hacer textos interesantes, es una cuestión de practicar y practicar y practicar y corregir y corregir y corregir.

\section{Para conocer más, puedes consultar:}

* Perla Mateo, M. P. (s.f.). Cómo redactar un artículo divulgativo. Recuperado de: https://comunicaciencia.unirioja.es/como redactar articulo divulgativo.shtml.

* Cacho Carranza, Y. (2 de marzo de 2016). ¿Cómo escribir un texto de divulgación científica? Recuperado de: http://www.cienciamx.com/index.php/ciencia/ humanidades/5803-compartir-el-conocimiento-cientifico-a-traves-de-ladivulgacion.

* Mocencahua, D. (23 de noviembre de 2016). 10 consejos para escribir divulgación científica. Recuperado de: http://radiobuap.com/2016/11/10-consejos-paraescribir-divulgacion-cientifical.

\section{Cómo citar este artículo}

* Murray-Tortarolo, Guillermo N. (2019). De cómo escribir un artículo de divulgación y no matar de aburrimiento tus lectores. Revista Digital Universitaria (RDU). Vol. 20, núm. 4 julio-agosto. Dol: http://doi.org/10.22201/codeic.16076079e.2019.v20n4. a4.

Recepción: 03/12/2018 Aprobación: 17/05/2019. 